\title{
The Impact of Web Browser Reader Views on Reading Speed and User Experience
}

\author{
Qisheng Li \\ University of Washington \\ Seattle, Washington \\ liqs@cs.washington.edu
}

\author{
Meredith Ringel Morris \\ Microsoft Research \\ Redmond, Washington \\ merrie@microsoft.com
}

\author{
Adam Fourney \\ Microsoft Research \\ Redmond, Washington \\ adamfo@microsoft.com
}

\author{
Kevin Larson \\ Microsoft \\ Redmond, Washington \\ kevlar@microsoft.com
}

\author{
Katharina Reinecke \\ University of Washington \\ Seattle, Washington \\ reinecke@cs.washginton.edu
}

\begin{abstract}
As reading increasingly shifts from paper to online media, many web browsers now provide a "Reader View," which modifies web page layout and design for better readability. However, research has yet to establish whether Reader Views are effective in improving readability and how they might change the user experience. We characterize how Mozilla Firefox's Reader View significantly reduces the visual complexity of websites by excluding menus, images, and content. We then conducted an online study with 391 participants (including 42 who self-reported having been diagnosed with dyslexia), showing that compared to standard websites the Reader View increased reading speed by $5 \%$ for readers on average, and significantly improved perceived readability and visual appeal. We suggest guidelines for the design of websites and browsers that better support people with varying reading skills.
\end{abstract}

\section{CCS CONCEPTS}

- Human-centered computing $\rightarrow$ Empirical studies in HCI; Accessibility;

\section{KEYWORDS}

Reader View; Online Reading; Dyslexia; Website Design

Permission to make digital or hard copies of all or part of this work for personal or classroom use is granted without fee provided that copies are not made or distributed for profit or commercial advantage and that copies bear this notice and the full citation on the first page. Copyrights for components of this work owned by others than the author(s) must be honored. Abstracting with credit is permitted. To copy otherwise, or republish, to post on servers or to redistribute to lists, requires prior specific permission and/or a fee. Request permissions from permissions@acm.org. CHI 2019, May 4-9, 2019, Glasgow, Scotland UK

(c) 2019 Copyright held by the owner/author(s). Publication rights licensed to ACM.

ACM ISBN 978-1-4503-5970-2/19/05_..\$15.00

https://doi.org/10.1145/3290605.3300754

\section{ACM Reference Format:}

Qisheng Li, Meredith Ringel Morris, Adam Fourney, Kevin Larson, and Katharina Reinecke. 2019. The Impact of Web Browser Reader Views on Reading Speed and User Experience. In CHI Conference on Human Factors in Computing Systems Proceedings (CHI 2019), May 4-9, 2019, Glasgow, Scotland UK. ACM, New York, NY, USA, 12 pages. https://doi.org/10.1145/3290605.3300754

\section{INTRODUCTION}

Reading information on screen and in web browsers has increasingly taken the place of traditional ways of reading. However, digital content can impede people's reading fluency and comfort due to visual clutter, advertisements, or a lack of contrast $[48,53]$. These problems are exacerbated for people with reading difficulties, such as dyslexia, which is a cognitive disorder that impacts people's reading ability in various ways. People with dyslexia ( 15-20\% of the world's population [2]) often have difficulties organizing language and eliminating non-relevant elements when finding and understanding information on websites [36].

Inspired by one of the strategies that some people with reading difficulties employ for better web readability - using the browser's Reader View [36] - this paper explores how Mozilla Firefox's Reader View affects people's reading performance and user experience. More specifically, we endeavor to answer the following research questions:

RQ1: When and how does Reader View change a webpage? RQ2: How does Reader View impact reading performance, perceived readability, and the user experience compared to the standard presentation of a website?

RQ3: Do people with dyslexia benefit more from the Reader View than those without?

The first research question (RQ1) was motivated by the fact that there is insufficient documentation on what the Reader View does for any of the popular browsers. This also means that website designers do not currently know how to design websites that could be transformed to Reader View pages. We 
therefore characterized which modifications are performed by the Reader View in the Firefox web browser [37] by inspecting its open-source code and by quantifying the difference in visual designs between standard webpages and their Reader View version. We show that Reader View is only triggered on $2 \%$ of homepages and $41 \%$ of their child pages in our sample of 1100 webpages, and that the low percentage is mainly due to insufficient word count in the main content section. We also show that the Reader View reduces both images and text by around a third, while doubling the use of uniformly colored areas, including white space. This results in significantly lower visual complexity and colorfulness compared to the original websites.

It is also important to empirically validate the utility of reading tools, given that they appear in most browsers now. To better understand the impact of the visual modifications that Reader View makes on people's reading performance and their subjective preferences (RQ2 \& RQ3), we conducted an online study with 391 participants (42 who self-reported having been diagnosed with dyslexia). We found that Firefox's Reader View had a significant effect on reading speed: The readers read content in Reader View 5\% faster than on standard websites. In addition, while dyslexic participants consistently rated the readability and user experience of webpages lower than participants without dyslexia, both groups rated the readability and aesthetics of the Reader View higher than the standard webpages. This suggests that the low visual complexity of Reader View websites benefits reading performance, perceived readability, and user experience.

\section{RELATED WORK}

Individual Differences In Reading Abilities. Differences in readers' skills and text comprehension are a result of multiple factors, such as working memory capacity [11], wordidentification and comprehension skills [21], and age, which was shown to negatively correlate with reading speed. For example, older subjects (aged 65-75) read significantly slower than younger subjects (aged 25-35) [40] with people's reading speed decreasing between 20 and 88 years of age from 103 to 76 words per minute (WPM) [50]. Lott et al. demonstrated a similar decline in reading speed between ages 58 to 102 [29].

People also experience reading difficulties due to cognitive disabilities. People with dyslexia, for example, have difficulties learning to read and spell and struggle to achieve the levels of reading fluency as their non-dyslexic peers [52]. Impairments with any stages of processing, including visual processing (e.g. [18, 22]), phonological and orthographic processing (e.g. [5, 28]), and semantic processing (e.g. [38, 51]) can cause difficulties with reading.
On-screen Readability. Screen reading has been found to be $10-30 \%$ slower than reading on paper [25]. While earlier studies (from the pre-tablet era) indicate that on-screen reading performance (measured by traditional metrics such as reading speed, accuracy and comprehension) lags behind paper [12], greater equivalence is being achieved now as computer technology rapidly develops and more sophisticated comparative measures are used (e.g. [24, 39]). However, text that is surrounded by images and advertisements, such as on websites, has been found to reduce reading performance compared to text without. The distraction by even static ads occurs through overt fixations toward ads rather than as covert processing of ads during reading [53].

While website readability has generally been a concern, people with dyslexia consistently rate websites as less readable than people without dyslexia [36]. They perceive websites as too dense and cluttered, with the choice of font type, font size, color, and contrast between colors impacting their ability to find information [36]. Fourney et al. further found that perceived readability is impacted by several lexical and aesthetic webpage features for people with dyslexia, such as average line length, ratio of text appearing in and out of sentences, and average image size [16].

Prior work has developed design guidelines for improving on-screen and website readability through better text presentation for average readers and people with dyslexia (see Table 1). For instance, Miniukovich et al. have developed 8 core design guidelines to improve website readability for people with dyslexia, such as by using larger fonts, narrower columns, and avoiding underlining and italics [35]. Many of these guidelines are based on prior work that evaluated how text readability is affected by font properties (e.g. [45, 47]), text styles (e.g. italics, underlining $[3,20]$ ), as well as character, line, and paragraph spacing (e.g. [6, 49]). Previous work also evaluated guidelines for average readers (see [14] for a review). However, due to inconsistencies in measurements and populations between studies with people with and without dyslexia, some of these guidelines are contradictory [14], and others lack rigorous evaluations and replications.

Technologies for Improving On-Screen Reading. A growing number of technologies have begun to emerge to improve on-screen reading. The Reader Views available in major web browsers are one example. Microsoft's Learning Tools additionally help users with reading challenges by offering features such as reading text aloud, highlighting text on screen word-by-word, and providing alternative spacing and fonts [34]. Similarly, the word processing software SeeWord provides users with some control over how information is displayed [17]. Many people with reading disabilities rely on tools that read a screen's content aloud (such as Dragon Naturally Speaking or even screen reader technologies designed 
Table 1: Design Guidelines that have been suggested to improve webpage readability for the average reader and for people with dyslexia. The column on the right indicates whether and how the Firefox Reader View applies these guidelines. ${ }^{*}$ [35] summarized previous research and suggested to avoid formatting texts in large-width columns, which contradicts the other two work cited.

\begin{tabular}{|c|c|c|c|c|}
\hline Guideline & Reference & Avg. Reader & $\begin{array}{l}\text { People with } \\
\text { Dyslexia }\end{array}$ & $\begin{array}{l}\text { Firefox } \\
\text { Reader View }\end{array}$ \\
\hline Use section headings to organize the content & [35] & & $\checkmark$ & $\checkmark$ \\
\hline Limit the amount of content on a page to avoid scrolling & [35] & $\checkmark$ & & $x$ \\
\hline Avoid using italics in the main body of the text & [35] & & $\checkmark$ & $\checkmark$ \\
\hline Avoid underlining large blocks of text & [35] & & $\checkmark$ & $\checkmark$ \\
\hline Use text size larger than $14 \mathrm{pt}$ & {$[35,47]$} & $\checkmark$ & $\checkmark$ & $\checkmark$ \\
\hline Use gray-scale foreground text and off-white background & {$[35,48]$} & $\checkmark$ & $\checkmark$ & $x$ \\
\hline Use a plain, evenly spaced sans serif font & {$[35,46]$} & $\checkmark$ & $\checkmark$ & $\checkmark$ \\
\hline Let the user change text, background colors, and enlarge text & [6] & $\checkmark$ & $\checkmark$ & $\checkmark$ \\
\hline *Increase the number of characters per line (contradict. findings) & {$[14,35,49]$} & $\checkmark$ & $\checkmark$ & $x$ \\
\hline Avoid unnecessary images, ads, and animations & {$[53]$} & $\checkmark$ & & $\checkmark$ \\
\hline Increase character spacing & {$[22,47,49]$} & $\checkmark$ & $\checkmark$ & $x$ \\
\hline Increase image size & {$[16]$} & $\checkmark$ & $\checkmark$ & $\checkmark$ \\
\hline
\end{tabular}

for people with visual impairments) [36]. While this solution is helpful to many users, audio speech is almost twice as slow than the reading speed of an average person [32]. Hence, improving reading rather than providing such workarounds is still advantageous.

\section{HOW “READER VIEW" CHANGES WEBSITES}

To answer our first research question, when and how a browser's Reader View changes a webpage, we analyzed the open-source implementation of Firefox Reader View [37]. We first characterized the steps Reader View takes to transform webpages by inspecting its source code, and then analyzed the visual changes Reader View triggers using a quantitative comparison of website image features. We selected Firefox Reader View because it is the only open-source implementation of the Reader View among major web browsers [1,33], which allows us to better understand any changes it triggers and enables reproducibility of our results. Note that our analysis focused on web browser Reader View of PC devices. We did not compare against mobile view because mobile websites usually contain different page layout, and people behave differently on mobile devices than on a PC.

\section{Characterizing the Reader View in Mozilla Firefox}

Mozilla provides an open-source, standalone version of the readability library used for Firefox Reader View ${ }^{1}$. The main file, Readability.js (obtained on 07/15/2018), transforms the original HTML Document to a structured, well-formatted one for better readability. Mozilla Firefox lets users toggle between Reader View and the original webpage by pressing a Reader View icon in the address bar. The address bar only

\footnotetext{
${ }^{1}$ https://github.com/mozilla/readability
}

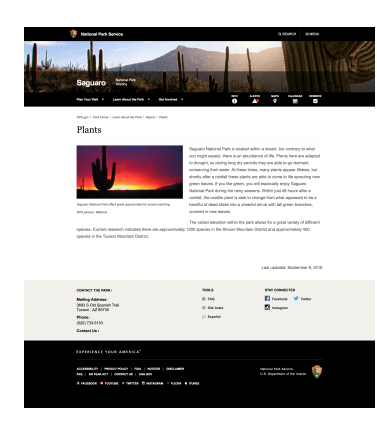

(a) Standard Webpage

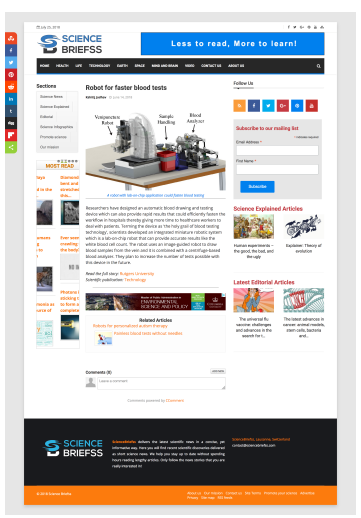

(c) Standard Webpage

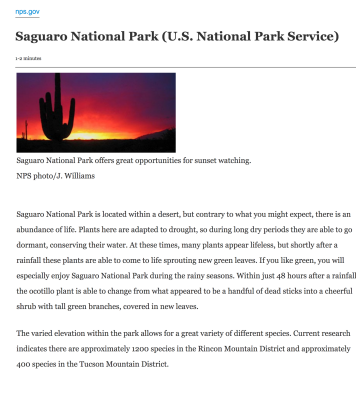

(b) Reader View

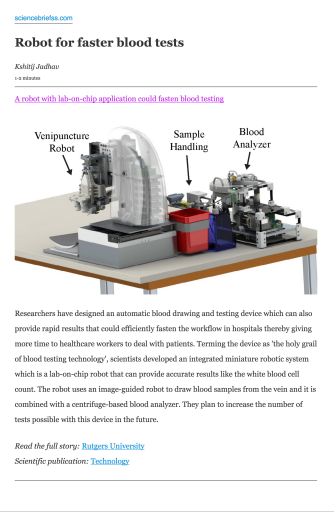

(d) Reader View
Figure 1: Two example webpages (a) \& (c) and how they are rendered in Firefox's Reader View (b) \& (d), respectively.

appears if a page has been determined to be transformable into Reader View, a process that we describe next.

Transformability Decision. Readability.js first determines whether a webpage has enough textual content to be transformed into its Reader View. The process begins by assembling a list of all 
document nodes that indicate paragraphs, pre-formatted text, or content divisions that contain line breaks (HTML nodes: $\langle p\rangle$, $\langle$ pre $\rangle$, or $\langle$ div $\rangle+\langle b r\rangle$, respectively). It then iterates over each node, adding the character length of the node's text content to an accumulator and skipping nodes unlikely to be content-bearing. Specifically, Readability.js skips nodes whose CSS class names or id attributes contain substrings such as "banner," "comment," or "header" - except in cases where the class names or ids also contain substrings such as "article," "body," or "main." Readability.js also skips nodes that contain certain list item configurations (HTML elements $\langle l i\rangle+\langle p\rangle$ ). If the accumulator reaches a value greater than 560 characters at any point, then the iteration terminates and Readability.js enables the Reader View button.

Content Decisions. If a webpage was deemed transformable into Reader View, the following steps are triggered to decide on the content that will be included:

(1) Parse an HTML string and build a JavaScript implementation of the document (DOM). Readability.js includes its own lightweight DOM parser.

(2) Prepare the DOM to be scraped, including stripping "script" and "style" tags, and handling bad markup by replacing two or more successive $\langle b r\rangle$ elements with a single $\langle p\rangle$, and replacing tags $<$ font $>$ to $<$ SPAN $>$.

(3) Extract the content that is most likely to be the main document content from the DOM tree using a variety of metrics, such as CSS class and id name (e.g., positive class names can be "content" and "main," unlikely candidates have names such as "menu," "sidebar," or "social"), element types (e.g., removing <tag> elements and nodes with empty text), and accumulated content score for each node (e.g., adding points for every 100 characters or for any commas within the paragraph) recursively added up from the child nodes in the DOM tree. Consequently, Reader View pages rarely contain menus, advertisements, logos, social sharing buttons, or sidebars, unless these elements are added to the HTML using different class and id names than what Readability.js looks for.

(4) Prepare the article node for display: Clean out elements such as iframes, textareas, buttons, single-cell tables, and elements that have more images than paragraphs. Then return the content wrapped up in a $\langle$ div $\rangle$.

(5) Finally, run any post-processing modifications to article content as necessary, for instance, fixing relative URLs and cleaning class attributes from every element except for the ones that Readability.js sets itself.

Style Decisions. With the content wrapped into one section $(<$ div $>$ ), Firefox renders the webpage using a predefined style sheet ${ }^{2}$. The default is a Serif font with font-size 20px,

\footnotetext{
${ }^{2}$ chrome://global/skin/aboutReader.css
}

content width of $660 \mathrm{px}$, a line-height of $35.2 \mathrm{px}$, and a white background. In addition to adding the Reader View icon to the address bar, which allows users to toggle between the original website and its Reader View, Firefox also provides a settings panel that let users adjust the text size, font, line spacing, and contrast in Reader View, or have a webpage read out loud. Users are able to choose either a Serif or Sans Serif font, choose either a light, dark, or sepia background color, and adjust the font size from 12px (pixel unit) to 28px, the max content width from $440 \mathrm{px}$ to $1420 \mathrm{px}$, and the line height from 22px to 57.2px. Note that in CSS the pixel unit does not correspond to a physical screen pixel; instead, it is the angular distance of a hypothetical pixel on a 96dpi screen at a distance of 28 inches $(71 \mathrm{~cm})$, which means that any style decisions made by Reader View render similarly on every screen [7]. As a result of these style decisions, Reader View pages are always one column, with images and text blocks stacked vertically on top of each other (Figure 1).

\section{Analysis of Reader View Availability}

To analyze how often Reader View finds webpages transformable, we randomly selected 100 websites from a dataset of website URLs obtained from the Alexa Top 500 Global Sites [19]. For each of the 100 websites, we selected 10 random child pages (one-level deep), for a total of 1100 page URLs. We then fed these URLs to Readability.js to evaluate if and how they might be transformed.

Results. Reader View was only available for $2(2 \%)$ homepages $^{3}$ and 406 (41\%) child pages from our sample. The low fraction of transformable homepages is because they often consist of little text and instead feature many visual elements, such as brand logos (individual images), navigation (lists), and calls to action (e.g., signup forms), without any sections containing a sufficient number of characters to trigger Reader View. We found that transformable webpages are mostly blogs, news, terms and conditions, or FAQs; in contrast, login pages, e-commerce sites, and pages with embedded content were unlikely to trigger Reader View. Interestingly, one of the homepages in our sample that Reader View did transform (https://www.vertbaudet.ch/) is an e-commerce site containing mostly images, which was transformed because it contained a sufficient number of characters in the elements at the bottom of the page.

\section{Quantitative Comparison of Visual Changes Between Standard Webpages and their Reader View Versions}

The previous section discussed when Reader View triggers changes, but did not yet describe concretely how it changes the visual appearance of websites. This section aims to quantify the changes between webpages and their Reader View.

\footnotetext{
${ }^{3}$ http://bagishared.com/ and https://www.vertbaudet.ch/
} 
Table 2: Comparison of image metrics between standard websites and their reader view versions. Image metrics were calculated using the VizWeb open-source library [31]. Significance levels: ${ }^{*} p<.05,{ }^{* *} p<.01,{ }^{* * *} p<.001$.

\begin{tabular}{|c|c|c|c|c|}
\hline Image Metric & Explanation & $\begin{array}{l}\text { Standard } \\
\text { Website } \\
\text { Mean (sd) }\end{array}$ & $\begin{array}{l}\text { Reader } \\
\text { View } \\
\text { Mean (sd) }\end{array}$ & $\begin{array}{l}\text { t-value } \\
(\mathrm{DF}=279)\end{array}$ \\
\hline Visual complexity [43] & A model of perceived website complexity (range 1-10) & $6.89(1.54)$ & $4.25(0.70)$ & $26.56^{* * *}$ \\
\hline Number of image areas & the number of image areas (adjacent images count as one) & $7.35(5.99)$ & $2.24(2.30)$ & $13.94^{* * *}$ \\
\hline Number of text groups & the number of horizontal groups of text characters & $21.45(12.92)$ & $8.15(2.61)$ & $17.23^{* * *}$ \\
\hline Colorfulness [43] & A model of perceived website colorfulness (range 1-10) & $3.97(1.29)$ & $2.40(0.58)$ & $20.95^{* * *}$ \\
\hline Saturation & the average pixel value in the HSV color space for saturation & $34.55(19.75)$ & $7.83(6.58)$ & $23.03^{* * *}$ \\
\hline Number of quadtree leaves & $\begin{array}{l}\text { Recursive division of a website screenshot into quadrants } \\
\text { (leaves) using color entropy as a criterion for further division }\end{array}$ & $73.73(45.73)$ & $37.84(8.65)$ & $13.06^{* * *}$ \\
\hline
\end{tabular}

To enable such statistical comparison between website designs, we analyzed the same 408 websites ( 2 homepages and 406 child pages) that we found could be transformed into Reader View in the section above. We first took screenshots of these webpages and their Reader View counterparts with a $1024 \times 1280$ resolution. We removed 94 webpage pairs where at least one page was less than 1280 pixels in height to ensure the same page dimensions for comparison. This resulted in 280 pairs of webpages for this analysis.

To quantify the visual design of standard webpages and the Reader View, we computed six image metrics (Table 2) for each screenshot using the algorithms provided by the open source project VizWeb [31]. We focused on image metrics that have been found to contribute to users' perceived visual appeal of a website as listed in [43] so as to characterize an important dimension of user experience. To analyze the difference between webpages and their Reader Views, we conducted paired t-tests and adjusted for multiple hypotheses testing using the Benjamini-Hochberg method [4].

Results. Our comparison shows that there are significant differences in the visual design of standard webpages and their Reader View counterparts (Table 2). Reader View pages are less colorful and less visually complex as shown in Figure 1's examples and supported by the statistics in Table 2 .

Design features that prominently contribute to the perception of visual complexity are the number of image and text areas [43]. On average, Reader Views have only two image areas (reduced from an average of seven) and eight text groups (down from 21). Reader View therefore reduces both images and text groups by around a third.

Correspondingly, Reader View websites are less colorful (per the computational model of colorfulness). This is mostly a result of two metrics, saturation and the number of quadtree leaves, which heavily influence perception of website colorfulness [43]. Indeed, Reader View websites have a significantly lower saturation value (reduced from an average of 34.55 to 7.83 ). The reduction in saturation is due to fewer images (i.e., fewer saturated pixels) and a uniform, white background.
Reader View webpages also avoid transitions between regions of different colors. This can be seen from the significant reduction of quadtree leaves from 73.73 to 37.84, suggesting that Reader View almost doubles the use of uniformly colored areas, including white space.

In summary, Reader View significantly reduces the number of images, text groups, the website's overall saturation, and any transitions between regions of different colors. As a result, Reader View pages are less visually complex and colorful than standard webpages. In combination, this might reduce distractions and support users in better focusing on the text - a hypothesis that we test in the next section.

\section{ONLINE EXPERIMENT}

We conducted an online study with the aim of evaluating how Firefox's Reader View impacts reading performance, perceived readability, and user experience (RQ2). The study also investigates how people with dyslexia benefit from the Reader View compared to those without (RQ3).

\section{Method}

The online experiment was developed as a 10-minute withinsubjects study (to account for individual differences in reading skills [44]) with two conditions, Standard Webpage vs. Reader View. The study was launched on the volunteer-based online experiment platform LabintheWild and advertised with the slogan "Test your reading speed!" on the site itself as well as on social media. After completing the study, participants received feedback on their reading speed in comparison to others. Providing this feedback rather than financially compensating participants was meant to attract intrinsically motivated volunteers who have been shown to exert more effort and provide more truthful responses than participants recruited through paid crowd platforms like Mechanical Turk [54].

The study was approved by our IRB, including a waiver of parental consent for minors participating in the study.

Reading Materials. To avoid learning effects from having seen the content of a webpage before, we constructed pairs of similar webpages, which had: 
(1) the same website category (e.g., news, e-commerce, etc.) with similar topics;

(2) a similar reading difficulty as measured by the FleschKincaid Grade Level [23] (a score indicating the U.S. grade level of education required to understand a given piece of text), with a difference less than 1 ;

(3) a comparable length measured by the word count in the title and main content (less than $20 \%$ difference);

(4) a similar visual design, as measured by having the same number of paragraphs and images.

Webpages were randomly selected from the Alexa Top 500 Global Sites dataset [19], and had to have a Reader View equivalent and use English text. To increase the likelihood that webpages were previously unknown to participants, we excluded those ranked 1-100 (i.e., the most popular ones). Because we had to compare participants' performance with standard webpages to their performance when using the Reader View of a different, but comparable, page (to avoid learning effects), we could not use the same sample from the quantitative analysis in Section 3 where the Reader View equivalents were not guaranteed to exist. Therefore, we manually explored the eligible websites and their child pages, created pairs, and modified the content of the pages by reducing the word count to keep the pairs of webpages comparable in length. We mitigated bias by randomly selecting all pages; those webpage pairs that were not comparable (according to the criteria listed above) were replaced by randomly selecting new webpages until a match was found.

The final stimuli were six webpages (comprising three pairs), of which we took screenshots of their standard view and their Reader View, resulting in 12 webpage screenshots (four examples are shown in Figure 1).

Metrics. We used the following metrics to gauge reading performance and user experience:

Reading speed: Calculated as word count divided by adjusted runtime in words per minute (WPM). Adjusted runtime measures how long participants spent on each webpage minus scrolling duration.

Comprehension questions: To check whether participants understood the content of the website and did not merely skim the text, we created three multiple-choice questions per webpage (e.g., "Where is the location of the film production?"). All questions could be answered directly from having read the text; the answers for at least two of the three questions were located in the first and the last paragraph to ensure the need to read the entire article. We tested the questions in a preliminary study with six participants.

Perceived Readability: Calculated based on 7 readability questions on a 7-point Likert scale $(1=$ strongly disagree, 7 = strongly agree) that we adapted from [36]. Participants were asked to rate their agreement with statements such as
"Major points were clearly stated" and "It was easy for me to lose my place while reading."

Aesthetics and User Experience: Participants were also asked to rate their level of agreement with 9 statements on a 7point Likert scale ( $1=$ strongly disagree, $7=$ strongly agree $)$ using Lavie and Tractinsky's aesthetics questionnaire [27], which subdivides an overall impression of aesthetics into classical aesthetics (e.g., "The webpage has a clean design.") and expressive aesthetics (e.g., "The webpage has a creative design"). The questionnaire is commonly used to evaluate the user experience of websites [30]. We removed one question, "The webpage uses special effects", from the expressive aesthetics scale because it is unlikely to be informative for a static webpage.

Relative subjective duration (RSD): Measures participants' perception of how long it took to read the webpage [10]. Participants were asked "Can you estimate how long you have spent on reading the webpage (shown below) in minutes?" and had choices in half-minute increments between 0.5 and 10 minutes. Previous work has shown that RSD not only predicts task engagement and task difficulty, but also aesthetic differences: the duration of difficult tasks or tasks with poor aesthetic qualities will be overestimated by participants while the duration of easy, aesthetically pleasing tasks tends to be underestimated [10, 26]. We hypothesize that if Reader View improves readability, then participants will underestimate the duration of reading in Reader View relative to the duration of reading the standard webpages.

Demographics: Participants were asked to self-report their age, gender, native language, level of education, whether they have dyslexia (using three options: "no", "yes, I have been diagnosed by a professional," and "yes, but I have not been formally diagnosed" as in $[16,36])$.

The 12 webpage screenshots and the list of associated comprehension questions and answers for each are provided as supplementary materials.

Procedure. The experiment began with a brief overview of the study, an informed consent form, and a voluntary demographic questionnaire, followed by the task's instructions.

The experiment was split into two parts, one for each condition (Reader View and Standard Webpage). The order of the two conditions was randomized across participants. Participants were shown 3 webpages per condition, for 6 trials in total. Participants were not told about these conditions. The order of webpages was randomized within each condition. Participants were asked to read each webpage word by word and to answer three required comprehension questions immediately after reading each webpage. At the end of each condition, participants were asked to answer the 7 readability questions, 9 user experience questions and 1 RSD question for the last webpage viewed in that condition. 
Table 3: Average subjective Likert scale measures on a 7-point scale by page condition (Standard Webpage vs. Reader View) and by dyslexia status (self-diagnosed and formally diagnosed dyslexics were grouped together because their ratings did not significantly differ). Mann-Whitney $U$ Tests were conducted to test whether Standard Webpage and Reader View received significantly different ratings, and whether participants with and without dyslexia provided significantly different ratings. Significant scales $(p<.05)$ are bolded.

\begin{tabular}{l|ccc|ccc|c}
\hline \multirow{2}{*}{ Likert Scale } & \multicolumn{3}{|c|}{ Standard Website } & \multicolumn{3}{c}{ Reader View } \\
& non-dyslexic & dyslexic & Cronbach's & non-dyslexic & dyslexic & Cronbach's & Significance \\
& $\mu(\mathrm{M})$ & $\mu(\mathrm{M})$ & alpha & $\mu(\mathrm{M})$ & $\mu(\mathrm{M})$ & alpha & $(p$ value $)$ \\
\hline Readability & $\mathbf{4 . 6 3 ( 5 )}$ & $\mathbf{3 . 7 5 ( 4 )}$ & .75 & $\mathbf{5 . 2 7}(\mathbf{6})$ & $\mathbf{4 . 5 3 ( 5 )}$ & .77 & $<.001$ \\
Classical Aesthetics & $\mathbf{4 . 1 1 ( 4 )}$ & $\mathbf{3 . 5 0 ( 4 )}$ & .91 & $\mathbf{5 . 0 0 ( 5 )}$ & $\mathbf{4 . 5 6 ( 5 )}$ & .84 & $<.001$ \\
Expressive Aesthetics & $2.93(3)$ & $2.63(2)$ & .85 & $2.83(3)$ & $2.91(3)$ & .86 & $=.39$ \\
\hline
\end{tabular}

A screenshot of the webpage was provided as a reminder. Participants were then given the opportunity to report on any technical difficulties, and to provide any other general comments or questions. The final page showed their personalized reading performance in comparison to others. The entire study took 10-12 minutes to complete.

Participants. The experiment was deployed online for 4 months and completed 428 times. We excluded 37 participants who self-reported participating more than once. Our analysis therefore reports on the data of 391 participants.

Participants were between 11-72 years old $(M=29.8, S D=12.5)$ and $55 \%$ were female. $69(18 \%)$ participants reported to have dyslexia, of which $42(61 \%)$ had been diagnosed by a professional, and 27 (39\%) had not been formally diagnosed. 286 (73\%) participants were English native speakers, while the native languages of other participants included 22 other languages. The plurality of participants $(41.9 \%)$ reported having completed college, $20.2 \%$ completed graduate school, and $22.0 \%$ high school. The remaining participants were enrolled in professional schools (6.4\%), pre-high school (2.6\%), or had finished a Ph.D. education (4.6\%).

\section{Analysis}

Reading Speed. We excluded 98 (of 2346) trials that were completed extremely fast (i.e., the reading speed was higher than median $+3 \times$ the Interquartile Range (IQR) (928 WPM)), indicating that participants might have only been skimming the text or not reading it at all. We also excluded one trial with a reading speed of less than $10 \mathrm{WPM}$. We further removed 363 trials where participants completed the study on a smartphone because people read and scroll differently on mobile devices than on a PC. To ensure that participants did not solely skim the text, we additionally discarded 111 trials where participants answered less than 2 out of 3 comprehension questions correctly.

We ran a series of linear mixed-effects regression models with (log-transformed) reading speed as the dependent variable and participant as a random variable. Fixed effects were page condition, dyslexia status (non-dyslexic, self-diagnosed, and diagnosed participants), the interaction between page condition and dyslexia status, as well as the control variables screenshot width, word count, age, and native English (i.e., whether a participant reported to be a native English speaker) as fixed-effect variables (Table 4). Variables were included based on a comparison of models using the Akaike information criterion (AIC). T-tests (p-values) were calculated using Satterthwaite approximations for degrees of freedom.

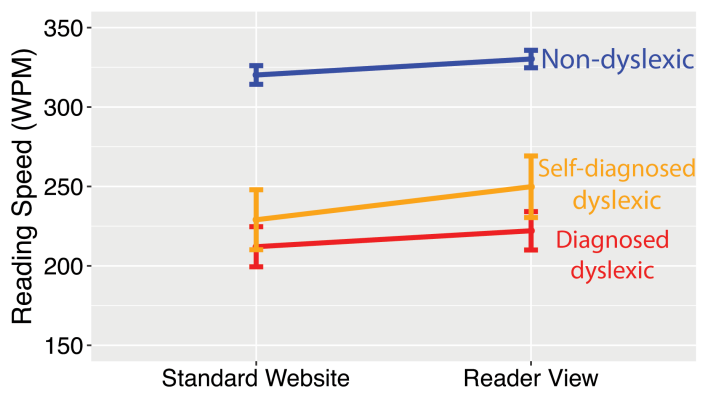

Figure 2: Average reading speed across non-dyslexic, diagnosed dyslexic, and self-diagnosed dyslexic participants in Words per Minute for Standard Webpages and their Reader Views. Error bars show standard error.

Subjective Ratings. For all Likert scale items, we tested internal consistency using Cronbach's alpha [9]. All scales showed high reliability with $\alpha>=.75$ (Table 3 ). We therefore used the averages of participants' responses for each scale.

The subjective ratings for readability and aesthetics were not normally distributed according to both visual inspection of histograms and Kolmogorov-Smirnov tests; hence, we conducted non-parametric Mann-Whitney $U$ tests for analysis. Mann-Whitney $U$ tests also showed that diagnosed and selfdiagnosed dyslexic participants did not provide significantly different ratings, leading us to group the two populations for the analysis of subjective questions.

Relative Subjective Duration (RSD). To analyze participants' perceptions of their reading duration, we first calculated the difference between participants' estimated and actual 
reading duration. We conducted an ANOVA comparing the duration difference between the two conditions. Page condition was modeled as a within-subject factor, dyslexia (nondyslexic, self-diagnosed, and diagnosed participants) as a between-subject factor, and a dyslexia by page condition interaction.

\section{Results}

Reading Speed. Our results show that, across all participants and for those trials where participants answered at least two of the three comprehension questions correctly, Reader View significantly increases reading speed by $5 \%$ compared to the standard webpages (see estimates in Table 4). People who reported having been formally diagnosed with dyslexia read significantly slower than non-dyslexic participants by $43.7 \%$ and they are also significantly slower than those who reported having dyslexia but who haven't been formally diagnosed.

We did not find a significant interaction effect between Reader View and Dyslexia, suggesting that all participants benefit from the Reader View at a similar rate (i.e., the slope of improvement between the two conditions is similar, albeit slightly steeper for self-diagnosed dyslexics). Non-dyslexic participants increased their reading speed from 320 to 330 WPM (college-educated adults have been previously found to have a reading speed of about 244 to $460 \mathrm{WPM}$ when reading on screen [13]), while self-diagnosed dyslexics and diagnosed dyslexics increased it from 229 to 250 WPM and 212 to 222 WPM, respectively (Figure 2).

Age, word count, and native language also significantly impacted reading speed. With every year of age, reading speed decreases by $1 \%$, confirming the findings of prior work [40]. Every additional word increases the reading speed by $0.1 \%$; and being a native English speaker significantly increases reading speed by $23 \%$. None of these factors interacted with Reader View, suggesting that Reader View does not differentially impact people of various ages or with different language backgrounds. Screenshot width also did not impact the reading speed.

Participants' comprehension scores did not differ between page conditions; instead, we found a speed-accuracy tradeoff with participants who read faster answered less questions correctly. This likely removes the effect of page condition on comprehension scores.

Subjective Ratings. As shown in Table 3 and Figure 3, participants rated the readability of Reader View pages significantly higher than standard websites (Figure 3a). They also felt that the Reader View had superior classical aesthetics (e.g., "clean," "pleasant") compared to standard webpages (Figure 3b), which suggests that the Reader View follows design rules that are thought to improve usability [27]. Ratings
Table 4: The results of a linear mixed-effect model predicting log reading speed.

\begin{tabular}{|l|rcrl|}
\hline Variable & Est. & SE & t-value & Pr(>|t|) \\
\hline (Intercept) & 5.593 & 0.17 & 32.85 & $<.001^{* * *}$ \\
Reader View [yes] & 0.048 & 0.01 & 3.60 & $<.001^{* * *}$ \\
Dyslexia [diagnosed] & -0.437 & 0.07 & -6.04 & $<.001^{* * *}$ \\
Dyslexia [self-diagnosed] & -0.371 & 0.10 & -3.86 & $<.001^{* * *}$ \\
RV $\times$ Dys [diagnosed] & -0.014 & 0.04 & -0.34 & $=.74$ (n.s.) \\
RV $\times$ Dys [self-diagnosed] & 0.073 & 0.06 & 1.31 & $=.19$ (n.s.) \\
Screenshot width & 0.00 & 0.00 & -0.56 & $=.58($ n.s.) \\
Word count & 0.001 & 0.00 & 24.96 & $<.001^{* * *}$ \\
Age & -0.010 & 0.00 & -6.05 & $<.001^{* * *}$ \\
Native English [yes] & 0.226 & 0.05 & 4.88 & $<.001^{* * *}$ \\
\hline
\end{tabular}

on expressive aesthetics (e.g., "fascinating," "creative") did not significantly differ between the conditions (Figure 3c). In contrast, we would have expected Reader View pages to receive lower ratings on expressive aesthetics than standard websites, given that the concept measures the creativity and originality of a design [27].

As Figure 3a shows, people with dyslexia rated webpages as significantly less readable compared to those without dyslexia in both conditions. They also provided lower ratings on classical aesthetics (Figure 3b), indicating that they perceive both standard and Reader View webpages as significantly less aesthetically pleasing (and, thus, usable [27]) than non-dyslexics.

Relative Subjective Duration (RSD). Participants overestimated their reading time by an average of $67 \mathrm{~s}(143 \%)$ in the Reader View and 64s (142\%) for Standard Webpages (the difference is not statistically significant, $F_{455}=0.1, p=0.75$ ).

Dyslexia had a significant main effect on the perceived reading duration $\left(F_{455}=4.65, p<0.05\right)$. A Welch's two sample t-test suggests that the standard webpages led people diagnosed with dyslexia and those without to similar overestimations of the reading duration (non-dyslexics mean = $63 \mathrm{~s}, \mathrm{sd}=85 \mathrm{~s}$, dyslexics mean $=107 \mathrm{~s}, \mathrm{sd}=92 \mathrm{~s}, t_{21}=2.01$, $p=.06)$. However, people formally diagnosed with dyslexia over-estimated the duration of the reading $(\mathrm{m}=104 \mathrm{~s}$, $\mathrm{sd}=$ $100 \mathrm{~s})$ more than people without $\left(\mathrm{m}=59 \mathrm{~s}, \mathrm{sd}=73 \mathrm{~s}, t_{29}=2.20\right.$, $p<.05)$ for the Reader View. Self-diagnosed participants did not differ from either non-dyslexics or people who had been formally diagnosed (Figure 4). There was no page condition by dyslexia interaction effect on RSD, meaning that participants' perceived task duration changed at similar rate between Standard Webpages and Reader Views.

\section{DISCUSSION}

Our work is the first to demonstrate that Firefox's Reader View converts websites into pages that significantly improve reading speed, perceived readability, and perceived classical aesthetics (suggesting that the design is perceived as 


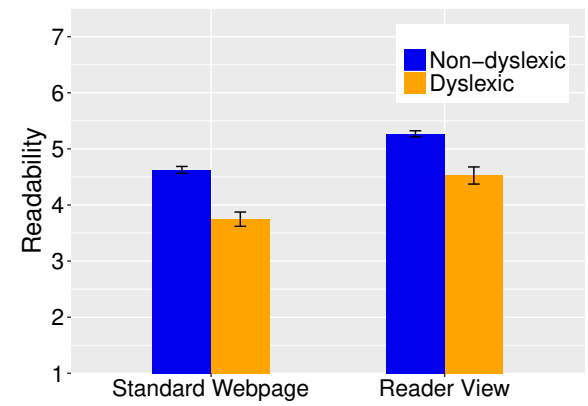

(a) Average perceived readability.

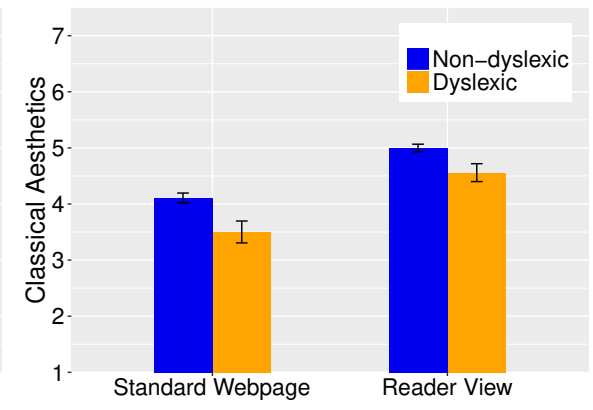

(b) Average classical aesthetics.

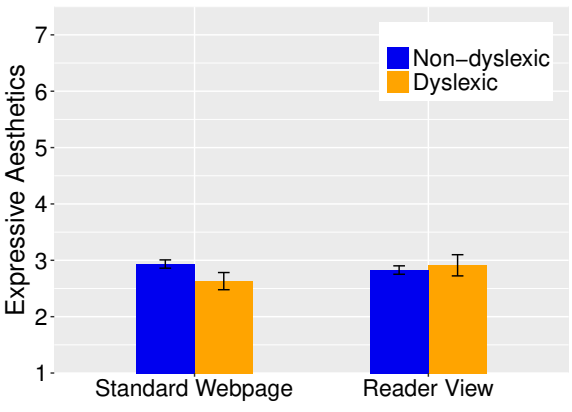

(c) Average expressive aesthetics.

Figure 3: Average ratings of perceived readability, classical and expressive aesthetics for standard and Reader View webpages by non-dyslexics and people with dyslexia (self-diagnosed and formally diagnosed dyslexics were grouped together because their ratings did not significantly differ). Error bars represent standard error.

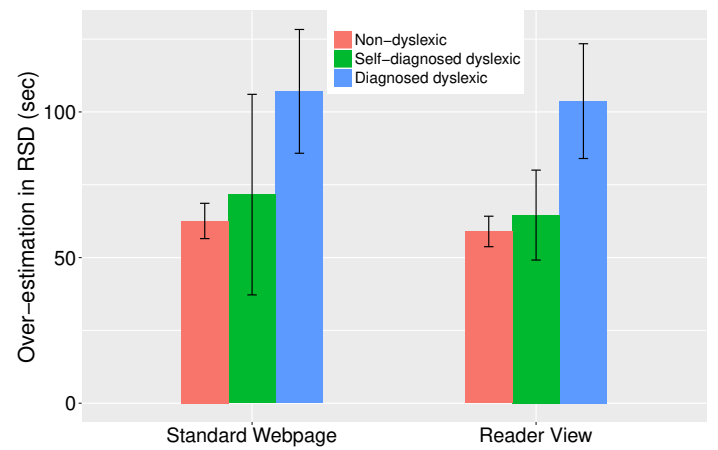

Figure 4: Over-estimation of participants' perception of reading duration $(\mathrm{RSD})$ relative to the actual time spent reading a webpage. Error bars represent the standard errors.

cleaner and more in line with recommendations for usable designs [27]). In particular, our participants improved their reading speed by $5 \%$ compared to standard websites on average. This is a modest, albeit significant improvement in reading speed: It is less than what speed reading training could achieve, for example, but is unlikely to impact a speedaccuracy trade-off that can result from speed reading [41]. However, we found that people with dyslexia did not improve their reading speed more from the Reader View than those without, and their overall reading speed remained well below that of non-dyslexic participants.

Our findings also showed that participants rated Reader View as significantly higher on classical aesthetics than standard webpages, while ratings on expressive aesthetics (a concept that measures the creativity and originality of a design [27]) did not significantly differ between Reader View and standard webpages. This suggests that Reader View conforms to usability-related design guidelines, such as being clean, clear, or symmetric [27], but its page design is not perceived as more sophisticated, fascinating, or creative than standard webpages. Hence, creatively designed websites might be valuable for an overall user experience, but detrimental for focused reading.

\section{Design Changes that Improve Reading}

Our work suggests that text-heavy websites ought to be designed with lower visual complexity than currently the case. Our analysis of the Reader View source code and quantitative comparison of visual changes that the Reader View triggers revealed what might cause the improvements in reading that our study showed: For one, Reader View reduces the number of text groups and images by a third. Its algorithm strips websites of any content deemed unnecessary for focused reading, including advertisements, menus, and logos. Images and text blocks are stacked on top of each other in a one-column layout rather than distributed across multiple columns as in many websites. Reader View also doubles areas that are uniformly colored, mostly by converting any background color to white space. As a result, Reader View pages are significantly less colorful and less visually complex than standard websites.

Previous studies have produced inconclusive results as to whether a one-column layout improves reading speed compared to a two- or three-column layout (see, e.g., [8] vs. [15]). However, most prior work suggests that longer line lengths, as measured by the number of characters per line, result in faster reading $[14,49]$. This suggests that Reader View's decision to use a larger content width than most standard websites plays a role in the reading speed improvement we have seen. It is also likely that Reader View's reduction of images contributes to an improved reading speed, since static images and ads distract reading [53]. Reader View's decision to display images and text in the same column means that users rarely see more than one image at once, which might have resulted in our participants being better able to avoid fixation on images [53].

\section{Should Reader View be the Standard View?}

We were surprised to find that only $2 \%$ of homepages and $41 \%$ of child pages (from a randomly selected sample of 100 website URLs) were available in Reader View. Our analysis of Reader View's source code showed that this is because 
homepages often do not contain a sufficient amount of text in a given section to trigger the Reader View. Websites that most often get converted into a Reader View are blogs and news websites, which usually have high word counts in the main content. However, due to the low availability of websites that do get transformed into Reader View, only few people benefit from the Reader View.

Given that we saw considerable improvements in reading speed, perceived readability, and classical aesthetics, one could argue that webpages with high word counts, such as blogs and news websites, should be presented in their Reader View by default, or even designed following the Reader View style sheet. This would mean that users are less likely to be exposed to advertisements (a change that would impact the revenue model of many websites) and less likely to be subjected to elements of branding. The latter could negatively affect a company's brand memorability, but also the users' orientation on the web since logos, color schemes, and other forms of branding can serve as navigational cues ("Am I still on the same website?"). Hence, defaulting to the Reader View (or equivalent design choices) might neither be feasible from a company's perspective, nor particularly beneficial to the user - unless they are in fact intending to read the content.

One design suggestion is that search engines could either indicate which pages offer Reader View (e.g., by including the icon next to the page in the result list), or allow a way for filtering for "readerable" pages. Users could then more easily benefit from Reader View pages; in addition, explicitly signaling Reader View availability could encourage developers to make their websites Reader View compatible.

\section{Designing for Reader View}

Our results provide insight into what triggers Reader View and what prevents a webpage from being transformed. This can help web designers and developers to know how to design more "readerable" websites. In addition, we found that Firefox's Reader View occasionally removes content that was essential for an article, or fails to remove content that is irrelevant. Our inspection of Firefox's Reader View source code revealed the tags that are used to determine what to include and exclude, which can support developers in creating websites that will trigger the Reader View (or avoid it). However, other major web browsers do not openly publish their source code and do not provide more than a light description of their Reader View features. This leaves web developers to guess what might or might not trigger the various implementations of the Reader View features in different browsers. While we hope that developers of the Reader Views in Edge, Safari, and other web browsers will provide better guidelines for developers to know what tags to include or exclude, we realize that openly revealing this information can also result in adversarial behavior. For example, developers might deliberately change tags to avoid advertisements from getting stripped out of the Reader View. To prevent this, companies could only reveal specific algorithmic rules, or they could provide tools that give developers feedback on their code without explicitly revealing any such rules.

\section{Additional Support for People with Dyslexia}

Our study showed that the Reader View improved the reading speed and perceived readability for participants with dyslexia. Perhaps most importantly, they perceived the readability of Reader View pages as equally high as non-dyslexic participants perceived the readability of standard websites. This indicates that the Reader View can be a step toward equalizing the reading experience between users with varying reading abilities, at least to some extent. However, Reader View does not bring people with dyslexia to the same level of performance as people without dyslexia. One finding that has been suggested by prior research as beneficial for people with dyslexia but that has not yet been leveraged in the Reader View is adding additional spacing between the letters within words [22]. This is helpful for those dyslexics who experience crowding problems-for them, the letters within words will appear jumbled. For them, adding additional spacing between the letters has led to an increase in reading speed and accuracy [22].

\section{LIMITATIONS AND FUTURE WORK}

Our study was not designed to disentangle the effect of specific design decisions on reading, but putting our results in context with prior work sheds light on which design changes that Reader View makes led to an improvement in reading speed. Future work could systematically explore which of the design changes result in the most noticeable improvement.

Another limitation of our study is that we only looked at websites as viewed on tablets, laptop or PC screens, but excluded mobile views and thus, smaller screen sizes. Websites that use a responsive layout often resemble the Reader View on a mobile screen, which is an interesting comparison for future studies.

Finally, our sample was not large and diverse enough to closely analyze the effects of demographics on perceived aesthetics and user experience that previous work has shown [42]. Therefore, the simple design inspired by the Reader View should be adopted with caution. We are excited to explore Reader View alternatives that improve both perceived aesthetics and reading speed over standard websites for people from various backgrounds.

\section{CONCLUSION}

This paper explored how Firefox's Reader View impacts reading fluency and user experience compared to standard 
websites. Our analysis of the source code and quantitative comparisons of Reader View's page designs with standard webpages showed that Reader View reduces the colorfulness and visual complexity of webpages, removing a third of images and text groups on average. An online study comparing Reader View with standard webpages showed that Reader View's design changes result in significant improvements in reading speed, perceived readability and aesthetics for people with and without dyslexia. However, we found that only $2 \%$ of homepages and $42 \%$ of child pages could be transformed into Reader View, suggesting that few websites provide this benefit. Our work is the first to systematically characterize how Reader View works, what improvements it achieves, and how this differs between people with varying reading abilities.

\section{DATASETS}

We make available the dataset used for quantifying visual differences between standard and Reader View websites, the dataset from our online study, and the R-code for analysis at https://github.com/QishengLi/CHI2019_Reader_View.

\section{ACKNOWLEDGMENTS}

This work was partially funded by NSF award 1651487 and by Microsoft. We thank our LabintheWild participants and the reviewers for their time and valuable suggestions.

\section{REFERENCES}

[1] Apple. 2018.

Working with Safari Reader. https: //developer.apple.com/library/archive/documentation/Tools/ Conceptual/SafariExtensionGuide/WorkingWiththeReader/ WorkingWiththeReader.html Accessed 15-September-2018.

[2] International Dyslexia Association. 2017. Dyslexia Basics. https: //dyslexiaida.org/dyslexia-basics Accessed 31-August-2018.

[3] Fakhrul Anuar Aziz and Husniza Husni. 2012. Interaction design for dyslexic children reading application: a guideline. (2012).

[4] Yoav Benjamini and Yosef Hochberg. 1995. Controlling the false discovery rate: a practical and powerful approach to multiple testing fournal of the Royal Statistical Society. Series B (Methodological) (1995), 289-300.

[5] Patricia Greig Bowers and Maryanne Wolf. 1993. Theoretical links among naming speed, precise timing mechanisms and orthographic skill in dyslexia. Reading and Writing 5, 1 (1993), 69-85.

[6] World Wide Web Consortium. 2008. Web Content Accessibility Guidelines (WCAG) 2.0. https://www.w3.org/TR/WCAG20/ Accessed 10-September-2018.

[7] World Wide Web Consortium. 2018. CSS Values and Units Module Level 4. https://www.w3.org/TR/css-values/\#px Accessed 10September-2018.

[8] Anthony Creed, Ian Dennis, and Stephen Newstead. 1987. Proofreading on VDUs. Behaviour \& Information Technology 6, 1 (1987), $3-13$.

[9] Lee J Cronbach. 1951. Coefficient alpha and the internal structure of tests. Psychometrika 16, 3 (1951), 297-334.

[10] Mary Czerwinski, Eric Horvitz, and Edward Cutrell. 2001. Subjective duration assessment: An implicit probe for software usability. In
Proceedings of IHM-HCI 2001 conference, Vol. 2. 167-170.

[11] Meredyth Daneman and Patricia A Carpenter. 1980. Individual differences in working memory and reading. fournal of Verbal Learning and Verbal Behavior 19, 4 (1980), 450-466.

[12] Andrew Dillon. 1992. Reading from paper versus screens: A critical review of the empirical literature. Ergonomics 35, 10 (1992), 1297-1326.

[13] Mary Dyson and Mark Haselgrove. 2000. The effects of reading speed and reading patterns on the understanding of text read from screen. Journal of Research in Reading 23, 2 (2000), 210-223.

[14] Mary C Dyson. 2004. How physical text layout affects reading from screen. Behaviour \& Information Technology 23, 6 (2004), 377-393.

[15] Mary C Dyson and Gary J Kipping. 1997. The legibility of screen formats: are three columns better than one? Computers \& Graphics 21, 6 (1997), 703-712.

[16] Adam Fourney, Meredith Ringel Morris, Abdullah Ali, and Laura Vonessen. 2018. Assessing the Readability of Web Search Results for Searchers with Dyslexia. In The 41st International ACM SIGIR Conference on Research and Development in Information Retrieval (SIGIR '18). ACM, New York, NY, USA, 1069-1072. https://doi.org/10.1145/ 3209978.3210072

[17] Peter Gregor, Anna Dickinson, Alison Macaffer, and Peter Andreasen. 2003. SeeWord - a personal word processing environment for dyslexic computer users. British fournal of Educational Technology 34, 3 (2003), 341-355.

[18] Stefan Hawelka and Heinz Wimmer. 2005. Impaired visual processing of multi-element arrays is associated with increased number of eye movements in dyslexic reading. Vision Research 45, 7 (2005), 855-863.

[19] Alexa Internet Inc. 2017. The Alexa Top Sites Services. http://www. alexa.com/topsites Accessed 15-August-2018.

[20] James E Jackson. 2014. Towards Universally Accessible Typography: A Review of Research on Dyslexia. (2014).

[21] Joseph R Jenkins, Lynn S Fuchs, Paul Van Den Broek, Christine Espin, and Stanley L Deno. 2003. Sources of individual differences in reading comprehension and reading fluency. Journal of Educational Psychology 95, 4 (2003), 719.

[22] Sung Jun Joo, Alex L White, Douglas J Strodtman, and Jason D Yeatman. 2018. Optimizing text for an individual's visual system: The contribution of visual crowding to reading difficulties. Cortex 103 (2018), 291-301.

[23] J Peter Kincaid, Robert P Fishburne Jr, Richard L Rogers, and Brad S Chissom. 1975. Derivation of new readability formulas (automated readability index, fog count and flesch reading ease formula) for navy enlisted personnel. (1975).

[24] Yiren Kong, Young Sik Seo, and Ling Zhai. 2018. Comparison of reading performance on screen and on paper: A meta-analysis. Computers \& Education 123 (2018), 138-149.

[25] Sri Hastuti Kurniawan and Panayiotis Zaphiris. 2001. Reading online or on paper: Which is faster? (2001).

[26] Kevin Larson and Rosalind Picard. 2005. The aesthetics of reading. In Appears in Human-Computer Interaction Consortium Conference, Snow Mountain Ranch, Fraser, Colorado.

[27] Talia Lavie and Noam Tractinsky. 2004. Assessing Dimensions of Perceived Visual Aesthetics of Web Sites. Int. F. Hum.-Comput. Stud. 60, 3 (March 2004), 269-298. https://doi.org/10.1016/j.ijhcs.2003.09.002

[28] Seija Leinonen, Kurt Müller, Paavo HT Leppänen, Mikko Aro, Timo Ahonen, and Heikki Lyytinen. 2001. Heterogeneity in adult dyslexic readers: Relating processing skills to the speed and accuracy of oral text reading. Reading and Writing 14, 3-4 (2001), 265-296.

[29] Lori A Lott, Marilyn E Schneck, Gunilla Haegerström-portnoy, John A Brabyn, Ginny L Gildengorin, Catherine G West, et al. 2001. Reading performance in older adults with good acuity. Optometry and Vision Science 78, 5 (2001), 316-324. 
[30] Sascha Mahlke. 2008. Visual aesthetics and the user experience. In The Study of Visual Aesthetics in Human-Computer Interaction (Dagstuhl Seminar Proceedings), Marc Hassenzahl, Gitte Lindgaard, Axel Platz, and Noam Tractinsky (Eds.). Schloss Dagstuhl - Leibniz-Zentrum fuer Informatik, Germany, Dagstuhl, Germany. http://drops.dagstuhl.de/ opus/volltexte/2008/1624

[31] Rahmatri Mardiko. 2018. Project VizWeb. https://bitbucket.org/ rmardiko/vizweb Accessed 11-September-2018.

[32] Ignatius G Mattingly, J Kavanagh, and I Mattingly. 1972. Reading, the linguistic process, and linguistic awareness. (1972).

[33] Microsoft. 2017. Reading View. https://docs.microsoft.com/en-us/ microsoft-edge/dev-guide/browser-features/reading-view Accessed 15-September-2018.

[34] Microsoft. 2018. Learning Tools. https://www.microsoft.com/en-us/ education/products/learning-tools Accessed 10-September-2018.

[35] Aliaksei Miniukovich, Antonella De Angeli, Simone Sulpizio, and Paola Venuti. 2017. Design Guidelines for Web Readability. In Proceedings of the 2017 Conference on Designing Interactive Systems (DIS '17). ACM, New York, NY, USA, 285-296. https://doi.org/10.1145/3064663.3064711

[36] Meredith Ringel Morris, Adam Fourney, Abdullah Ali, and Laura Vonessen. 2018. Understanding the Needs of Searchers with Dyslexia. In Proceedings of the 2018 CHI Conference on Human Factors in Computing Systems (CHI '18). ACM, New York, NY, USA, Article 35, 12 pages. https://doi.org/10.1145/3173574.3173609

[37] Mozilla. 2018. Firefox Reader View for Clutter-free Web Pages. https://support.mozilla.org/en-US/kb/ firefox-reader-view-clutter-free-web-pages Accessed 23-June2018.

[38] Kate Nation and Margaret J Snowling. 1998. Semantic processing and the development of word-recognition skills: Evidence from children with reading comprehension difficulties. fournal of Memory and Language 39, 1 (1998), 85-101.

[39] Jan M Noyes and Kate J Garland. 2008. Computer- vs. paper-based tasks: Are they equivalent? Ergonomics 51, 9 (2008), 1352-1375.

[40] Lorraine A Ramig. 1983. Effects of physiological aging on speaking and reading rates. Journal of communication disorders 16, 3 (1983), 217-226.

[41] Keith Rayner, Elizabeth R Schotter, Michael EJ Masson, Mary C Potter, and Rebecca Treiman. 2016. So much to read, so little time: How do we read, and can speed reading help? Psychological Science in the Public Interest 17, 1 (2016), 4-34.

[42] Katharina Reinecke and Krzysztof Z. Gajos. 2014. Quantifying Visual Preferences Around the World. In Proceedings of the SIGCHI Conference on Human Factors in Computing Systems (CHI '14). ACM, New York, NY, USA, 11-20. https://doi.org/10.1145/2556288.2557052

[43] Katharina Reinecke, Tom Yeh, Luke Miratrix, Rahmatri Mardiko, Yuechen Zhao, Jenny Liu, and Krzysztof Z. Gajos. 2013. Predicting Users' First Impressions of Website Aesthetics with a Quantification of Perceived Visual Complexity and Colorfulness. In Proceedings of the SIGCHI Conference on Human Factors in Computing Systems (CHI '13). ACM, New York, NY, USA, 2049-2058. https: //doi.org/10.1145/2470654.2481281

[44] Luz Rello. 2015. Dyslexia and Web Accessibility: Synergies and Challenges. In Proceedings of the 12th Web for All Conference (W4A '15). ACM, New York, NY, USA, Article 9, 4 pages. https://doi.org/10.1145/ 2745555.2746655

[45] Luz Rello and Ricardo Baeza-Yates. 2013. Good Fonts for Dyslexia. In Proceedings of the 15th International ACM SIGACCESS Conference on Computers and Accessibility (ASSETS '13). ACM, New York, NY, USA, Article 14, 8 pages. https://doi.org/10.1145/2513383.2513447

[46] Luz Rello and Ricardo Baeza-Yates. 2016. The Effect of Font Type on Screen Readability by People with Dyslexia. ACM Trans. Access.
Comput. 8, 4, Article 15 (May 2016), 33 pages. https://doi.org/10.1145/ 2897736

[47] Luz Rello and Ricardo Baeza-Yates. 2017. How to Present More Readable Text for People with Dyslexia. Univers. Access Inf. Soc. 16, 1 (March 2017), 29-49. https://doi.org/10.1007/s10209-015-0438-8

[48] Luz Rello and Jeffrey P. Bigham. 2017. Good Background Colors for Readers: A Study of People with and Without Dyslexia. In Proceedings of the 19th International ACM SIGACCESS Conference on Computers and Accessibility (ASSETS '17). ACM, New York, NY, USA, 72-80. https: //doi.org/10.1145/3132525.3132546

[49] Luz Rello, Gaurang Kanvinde, and Ricardo Baeza-Yates. 2012. Layout Guidelines for Web Text and a Web Service to Improve Accessibility for Dyslexics. In Proceedings of the International Cross-Disciplinary Conference on Web Accessibility (W4A '12). ACM, New York, NY, USA, Article 36, 9 pages. https://doi.org/10.1145/2207016.2207048

[50] Claudia Rodríguez-Aranda. 2003. Reduced writing and reading speed and age-related changes in verbal fluency tasks. The Clinical Neuropsychologist 17, 2 (2003), 203-215.

[51] Enrico Schulz, Urs Maurer, Sanne van der Mark, Kerstin Bucher, Silvia Brem, Ernst Martin, and Daniel Brandeis. 2008. Impaired semantic processing during sentence reading in children with dyslexia: combined fMRI and ERP evidence. Neuroimage 41, 1 (2008), 153-168.

[52] Sally E Shaywitz. 1998. Dyslexia. New England fournal of Medicine 338, 5 (1998), 307-312.

[53] Jaana Simola, Jarmo Kuisma, Anssi Öörni, Liisa Uusitalo, and Jukka Hyönä. 2011. The impact of salient advertisements on reading and attention on web pages. Journal of Experimental Psychology: Applied 17, 2 (2011), 174.

[54] Teng Ye, Katharina Reinecke, and Lionel P Robert Jr. 2017. Personalized Feedback Versus Money: The Effect on Reliability of Subjective Data in Online Experimental Platforms. In Companion of the 2017 ACM Conference on Computer Supported Cooperative Work and Social Computing. ACM, 343-346. 